\title{
Le nouvel examen fédéral, pour «de faux»?
}

\section{Raphaël Bonvin}

Dr, Unité pédagogique, Faculté de biologie et de médecine, CHUV Lausanne

L’article de Monsieur Cortessis présente le vécu du travail effectué par un patient standardisé participant au nouvel examen fédéral mis en place dès 2011 [1] qui est venu remplacer l'examen en place depuis plusieurs décennies. Cet examen apparaît dans un contexte de changement profond des études de médecine entre la réforme Bologne et la nouvelle loi sur les professions médicales ou LPmed (loi votée en 2006 remplaçant l'ancienne datant de 1877!) [2]. Aujourd'hui, les étudiants terminent leur formation prégraduée avec un diplôme de Maîtrise en Médecine délivré par l'Université. Ce n'est qu'en possession de cette Maîtrise qu'ils sont admis à l'examen fédéral de médecine, dont la réussite leur ouvrira les portes de leur formation médicale postgraduée et leur permettra de travailler en clinique sous supervision. En quelque sorte, les études de médecine se terminent avec la Maîtrise universitaire, et la formation postgraduée commence avec l'examen fédéral.
Ce nouvel examen fédéral comporte deux parties. Un examen écrit avec deux sessions à 150 questions QCM, et un examen pratique de type ECOS (pour Examen Clinique Objectif Structuré). Cet examen repose sur la participation de patients standardisés dont vous pouvez lire l'excellent témoignage de Monsieur Cortessi. Au cours de cet examen, les candidats rencontrent des patients présentant différents tableaux cliniques issus tant de la médecine ambulatoire

Le nouvel examen met justement l'accent sur le comportement du futur médecin avec son patient.

qu'hospitalière. Ils disposent d'un temps fixe, en l'occurrence 15 minutes, pour effectuer une anamnèse et un examen clinique ciblés ainsi que pour proposer une prise en charge. Leur performance est évaluée par les examinateurs qui disposent d'une grille d'observation; les examinateurs n'interagissent généralement pas avec les candidats, ne les questionnent pas,

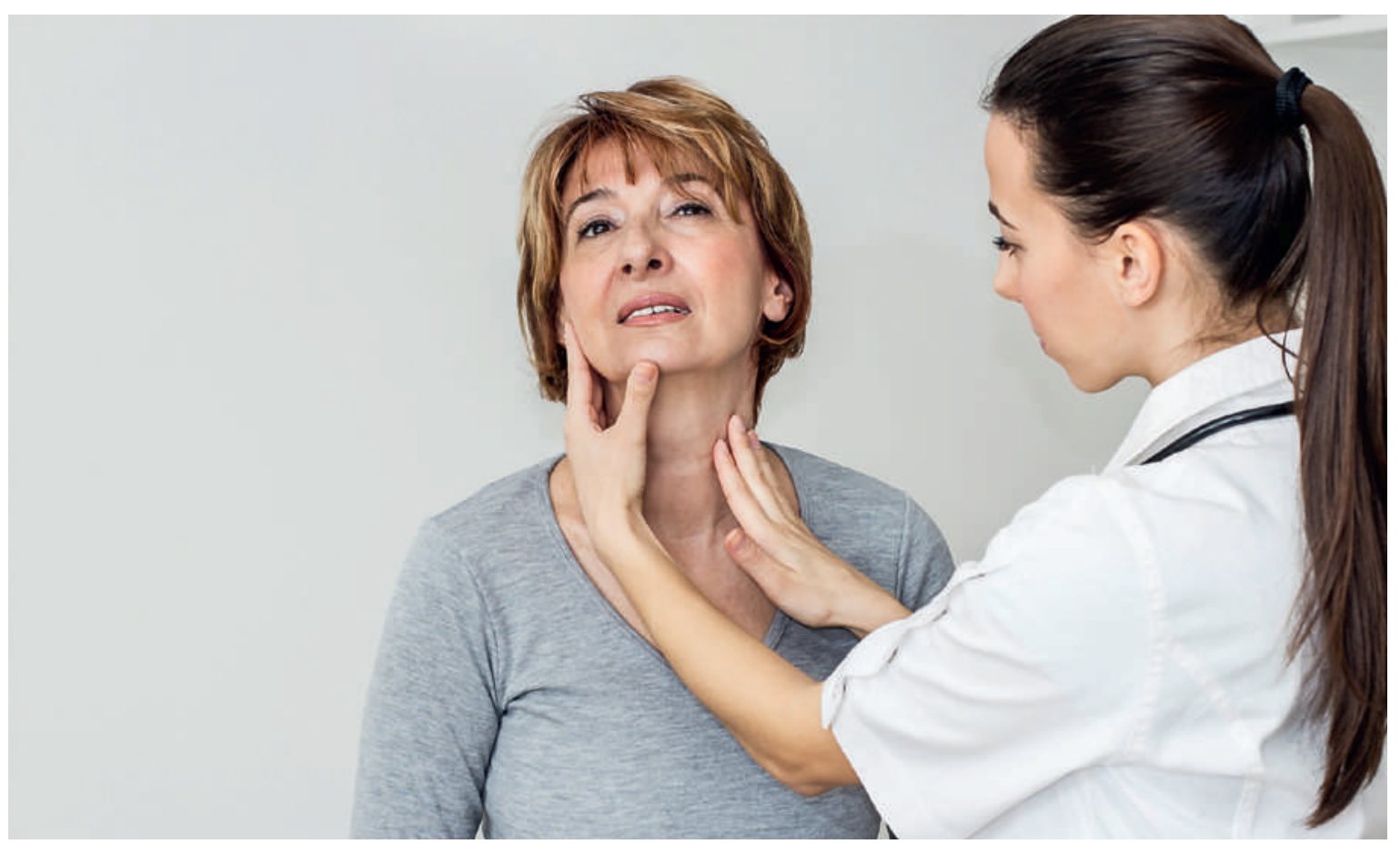

Avec des vrais patients on ne pouvait pas tester certaines situations cliniques comme des situations d'urgence ou des premières consultations. 
mais observent leur comportement avec le patient. Concrètement, tous ces changements font que les nouveaux médecins ne sont plus testés avec de vrais patients, mais avec des patients standardisés. Cela peut surprendre et même choquer. Mais laissons passer la première vague d'émotion et regardons déjà ce que nous gagnons avec cette approche. Lors des 9 examens oraux que la plupart d'entre nous avons dû passer sous l'ancienne ordonnance fédérale, nous étions effectivement confrontés à de vrais patients, le plus souvent selon le scénario suivant: le candidat allait examiner seul son patient dans sa chambre et après un temps d'attente plus ou moins long, présentait et discutait le cas (rarement le patient) lors de l'examen oral avec les examinateurs. L'accent était généralement mis sur les connaissances mais rarement sur le comportement avec le patient et la manière de réaliser l'anamnèse et l'examen physique et encore moins sur la manière de communiquer avec ce dernier (vu que le patient était vu en absence des examinateurs). Le nouvel examen met justement l'accent sur le comportement du futur médecin avec son patient, sur la prise d'anamnèse et l'examen physique, ainsi que la prise en charge etc., considérant que les connaissances sont testées par le QCM. L'approche par simulation permet également de tester des situations cliniques qui ne pouvaient être testées avant comme des situations d'urgence, des premières consultations (des patients voyant le médecin pour la première fois), des situations mal définies et instables, ou des situations relationnelles difficiles (refus d'un traitement, etc.). Un autre avantage de ce nouvel examen est sa structure en tableaux cliniques prédéfinis, de patients entraînés à jouer un scénario donné et de grilles d'observation. Cela favorise une évaluation bien plus équitable et comparable que les examens oraux. Tous les candidats de Suisse testés le même jour sont exposés aux mêmes 12 situations cliniques et évalués avec les mêmes critères d'observation. L'effet de la note à la "tête du client» (effet parfois réel, parfois imaginé) est fortement diminué. Fini le temps où la note était quasiment connue en découvrant quel expert allait nous examiner. Et fini également les grandes différences de résultats entre les facultés. Depuis son introduction, les résultats de l'examen fédéral des 5 facultés sont très proches et ne montrent pas de différences significatives.

Evidemment, cette approche présente également des désavantages. Pour les examinateurs, l'exercice peut être frustrant: le désir d'en savoir plus sur le raisonnement motivant un candidat d'agir d'une certaine manière est presque impérieux, mais l'examinateur doit se limiter à l'observation. La simulation, pour sa part, a également ses limites empêchant certaines situations d'être (bien) testées comme en pédiatrie. De plus, l'examen pratique perd en authenticité par rapport à l'examen d'un vrai patient. Si le candidat ne se prête pas au jeu (n'entre pas dans la simulation), alors faire un examen "pour de faux" chez un "malade» en bonne santé peut être vécu comme ridicule. Cette question d'authenticité n'est d'ailleurs pas tant un problème de qualité du jeu des patients standardisés. Ceux-ci passent en effet inaperçus aux yeux de cliniciens expérimentés dans le cadre de projets de recherche où, en tant que patients «incognito», ils sont

Depuis son introduction, les résultats de l'examen fédéral des 5 facultés sont très proches.

invités à consulter pour évaluer certains aspects de la prise en charge - précisons que les médecins participant à ces études ont donné leur accord, savent que des patients «incognito» consulteront, mais ne savent pas quand. C'est donc bien plus une question d'anticipation (sachant qu'il s'agit d'un "faux" patient) et de contexte, certainement exacerbé par le stress de l'événement, qui peuvent rendre une telle rencontre artificielle. Paradoxalement, cette approche cherche à mettre le travail avec le patient au centre de l'attention, en donnant une importance primordiale à l'interaction avec celui-ci, à la mise en pratique des connaissances en présence du patient, à l'exercice du métier de l'anamnèse et de l'examen clinique.

Introduit d'abord au Canada puis aux Etats Unis pour leur examen de licence (équivalent de notre examen fédéral), cette manière de tester la pratique clinique par un ECOS représente aujourd'hui la référence pour un examen fiable et équitable et un complément nécessaire aux QCM. En plus d'offrir un examen plus objectif et équitable, cet examen pratique permet surtout de valoriser et de renforcer la formation pratique (séméiologie, communication, prise en charge clinique) dans l’enseignement prégradué.

\section{Références}

1 Guttormsen S, Beyeler C, Bonvin R, Feller S, Schirlo C, Schnabel K, Berendonk C. The new licensing examination for human medicine: from concept to implementation. Swiss Med Wkly. 2013;143:w13897.

2 Brunet L, Monti M, Bonvin R. Le médecin compétent au XXI siècle et pourquoi les études de médecine ont changé. Revue Médicale Suisse. 2014;448(10):2048-52. 